\title{
Helicobacter pylori and eye diseases in an Iranian hospital
}

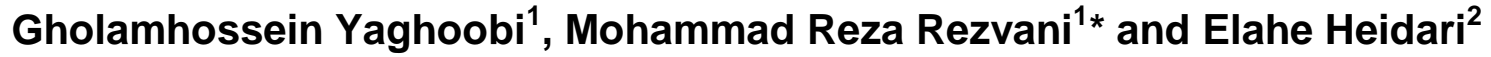 \\ ${ }^{1}$ Birjand University of Medical sciences, Ophthalmology Department, Valiasr Hospital, Southern Khorasan, Social \\ Determinant Health Research center, Birjand, Iran. \\ ${ }^{2}$ Mashad University of Medical Science, Pediatrics ward, Emmam Reza Hospital, Mashad, Iran.
}

Accepted 26 April, 2013

\begin{abstract}
There is evidence of Helicobacter pylori infection that induces eye diseases. This study was done to find if there is any association between $\boldsymbol{H}$. Pylori and ocular diseases, and if there is any difference in color of eye among them. Of all the 35 patients tested for biopsy with positive $\mathrm{H}$. pylori, $23(65.7 \%)$ were females and $12(34.3 \%)$, males. The mean age of patients was $39.65 \pm 18.55$ years. $20(57.1 \%)$ patients were below 40 years old; $8(22.9 \%)$ patients, 40 to 60 years and only 7 patients were more than 60 years old. Most of the patients were 20 years old and had no ophthalmic or systemic diseases in juvenile age group; most of them with the best correct vision were within normal measure, except the old patients who had normal tension glaucoma and two cataract cases. Brown eyes were the commonest but blue color was the least without any risk of ocular diseases or $\boldsymbol{H}$. Pylori associated with more prevalent brown iris color in study group. Ophthalmic extragastric diseases do not show any association between eye diseases and $\boldsymbol{H}$. pylori infection in juvenile age groups. Pesudoexfolitive glaucoma and two cases of cataract were among the old age related diseases which may be due to the late appearance of $\boldsymbol{H}$. pylori effect. Best visual acuity, cup/disc character and brown color were the most associated finding.
\end{abstract}

Key words: Helicobacter pylori, extragastric manifestations, ophthalmic diseases.

\section{INTRODUCTION}

There is increasing evidence that Helicobacter pylori $(H$. pylori) is an important pathogen in human infections. It is confined to the stomach and induces a strong systemic immune host response. Therefore, plausible untoward effects of these responses may contribute to the development of disease in areas other than gastrointestinal tract (Feghhi et al., 2008). A possible association between $H$. pylori infection and eye diseases, including Sjogren syndrome, blepharitis, central serous chorioretinopathy and uveitis has been proposed (Izzotti_et al., 2009). H pylori plays a potential role in the pathophysiology of many neurological and ophthalmological disorders also (cerebrovascular diseases, migraine, Alzheimer's disease, epilepsy, Parkinson's disease, multiple sclerosis, peripheral neuropathies, glaucoma, non- arteritic anterior ischemic optic neuropathy and lung cancer) (Deng et al., 2013).

H. pylori-specific IgG antibody levels significantly increase in the aqueous humor and serum of patients with primary open angle glaucoma (POAG) and Pesudoexfoliation glaucoma (XFG). These findings support a role of $\mathrm{H}$. pylori infection in the pathobiology of glaucoma (Jannis et al., 2003).

Neither $H$. pylori infection nor seropositivity for virulent CagA-bearing $H$. pylori strains has significant association with the occurrence of glaucoma of any type (Kurtz et al., 2008). Eye color is one of the major racial and diagnostic features in anthropological studies and it is used to characterize differences between populations where certain authors have shown some associations between eye color 
Table 1. Patients' demography.

\begin{tabular}{ll}
\hline Parameter & Value \\
\hline Female & $23(65.7 \%)$ \\
Male & $12(34.3 \%)$ \\
Age $40 y \geq$ & $20(57.1 \%)$ \\
Age 40 y> & $15(42.8 \%)$ \\
Y=year & \\
\hline
\end{tabular}
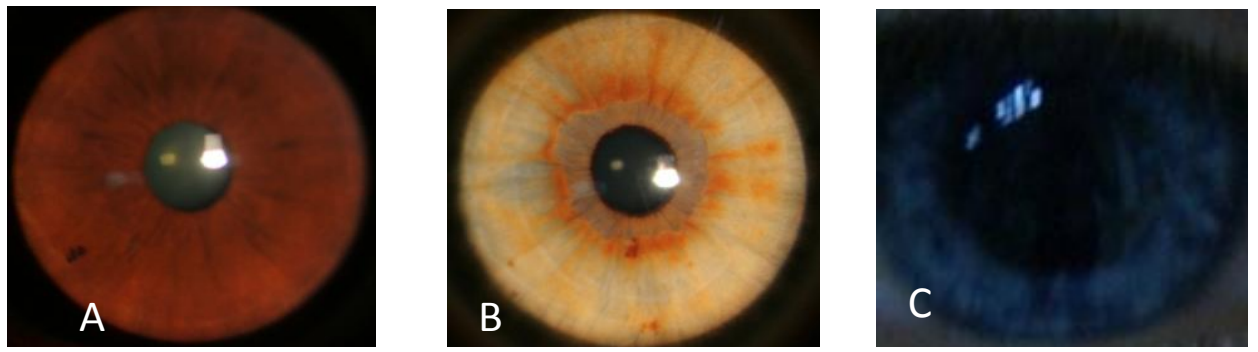

Figure 1. Iris colour classification; sample, $A=$ brown, $B=$ Green, $C=B l u e$.

and the constitutional type (Dzintra and Vētra, 2011). Farooq et al. 's research demonstrated that current data are very limited for establishing any causal relationship between $H$. pylori and most of the above mentioned disorders (Farooq and Bhatt, 2008).

The possibility of long-term effects of $H$. pylori eradication therapy on the cause of these disorders could be a preventive parameter of human discomfort if this relationship could be established. So, this study was done to find extragastric eye diseases in biopsy established $H$. pylori infection.

\section{MATERIALS AND METHODS}

Thirty-five (35) subjects who participated in this study were recruited from 1st March, 2011 to 31 st December, 2011 from the Department of Ophthalmology. An ethics committee approval was obtained from the Institutional Review Board. Participants were enrolled after signing the consent form. Patients who had indication of endoscopic evaluation due to gastric disorders or peptic ulcer were enrolled in this study. Diagnostic approach used in this study was Rapid Urea Test (RUT) for all biopsy specimen of $H$. Pylori infection.

All of the 35 patients were made to complete ophthalmic examinations, including a slit-lamp examination, intraocular pressure (IOP) measurement with a Goldman applanation tonometer and fundus and optic disc examinations, using a $90 \mathrm{D}$ biomicropscopy for iris color detection. Diagnosis of glaucoma was established based on IOP exceeding $29 \mathrm{mmHg}$ and optic disc character at the initial visit or visual field examinations using a Humphrey visual field analyzer (Carl zeiss meditec, Dubin, CA, USA) to define suspected cases.

\section{RESULTS AND DISCUSSION}

Of the 35 patients, $23(65.7 \%)$ were females and 12 $(34.3 \%)$, males. The mean age of the patients was 39.65 \pm 18.55 years. $20(57.1 \%)$ patients were below 40 years old; $8(22.9 \%)$ patients, 40 to 60 years and only 7 patients were more than 60 years (Table 1 ).

Majority of our patients (28) had no other systemic diseases except 7 (20\%) who had history of ischemic heart diseases (two cases), asthema (one case), hypertensions (two cases) and anemia (two cases). Ophthalmic examinations including visual acuity were good in $80 \%$ of cases. Biomicroscopy of anterior segment and IOP mean $(14.38 \pm 4.37 \mathrm{~m} \mathrm{Hg})$ were within normal limits except one case of pseudoexfoliative glaucoma and two cases of cataract. There were no optic nerve or retinal diseases in these groups of 35 subjects with positive cases of $\mathrm{H}$. Pylori. We could not find any other ocular inflammation or eye disease other than the ones mentioned above.

As shown in Figure 1, the commonest iris color was brown in $20(57.1 \%)$, green iris color in $13(37.1 \%)$ and 2 $(5.7 \%)$ had blue eye (Table 2$)$.

This study shows that $H$. pylori infection is associated with two cases of cataract and one case of pseudoexfoliative glaucoma. The 3 patients that had ocular diseases are old and it could be related to other possible risk factors other than $H$. pylori infection or may be a long time effect of $H$. Pylori infection. Our limitation in this study is descriptive and lacks control group because it is not logical to do biopsy in normal subject or do comparisons with other diagnostic approaches. There are studies that indicated ophthalmic diseases (Sergio et al., 2006; Kountouras_et al., 2011; Choi et al., 2010). A report by Banić et al. (2012) among several diseases are associated with $H$. pylori infection; some of these, such as cardiovascular disease and open angle glaucoma have consistent evidence of a causative role; while for others, further studies are needed to verify the association. There 
Table 2. Distribution of Ophthalmic manifestation and associated systemic diseases.

\begin{tabular}{llc}
\hline & \multicolumn{1}{c}{ Variable } & Number (\%) \\
\hline & Visual acuity more than $20 / 30$ & $80 \%$ \\
& Mean intraocular pressure $(\mathrm{mg} \mathrm{Hg})$ & $14 \mathrm{~m} 38 \pm 4.38$ \\
& Glaucoma & 1 \\
& Pseudo exfoliation & 1 \\
Ophthalmic & Retinal diseases & - \\
manifestation & Optic nerve diseases & - \\
& Intraocular inflammation & - \\
& Brown iris color & $20(50.17 \%)$ \\
& Green iris color & $13(37.17 \%)$ \\
Associated systemic & $2(5.7 \%)$ \\
diseases & Blue iris color & 2 \\
& Ischemic heart diseases & 1 \\
& Asthema & 2 \\
& Hypertention & 2 \\
\hline
\end{tabular}

is correlation between the iris color and certain conditions such as cataract and AMD with lighter eye colors. Several somatic or hereditary disorders can easily be recognized by the characteristic changes of the iris by just looking at it or the overlying cornea or intervening lens (Hashemi et al., 2010; Morrison, 2010).

We use the iris color to illustrate how recognition of some common and rare diseases that can be identified by direct visualization in the outpatient clinic can lead to prevention and management of underlying complications. The ocular diseases of our study were associated with brown color which was the commonest iris color 20 $(57.1 \%)$ versus green iris color in $13(37.1 \%)$ and 2 $(5.7 \%)$ blue eye. The best approach to control these diseases is easy and cheap accessibility which can help to eradicate human discomfort. But to elucidate the degree $H$. pylori gets involved in the development of eye diseases and how to eradicate it as well as its cost, there will be need for future studies to clearly adjust various factors for comparing this infectious agent and other extra gastric diseases.

This descriptive study could not find any ophthalmic diseases among $H$.pylori positive of less than 60 years old patients. As there is a practical approach to treat $\mathrm{H}$. Pylori infection, any of its casual in relation to extragastric diseases could also be prevented. Pesudoexfoliative glaucoma in one elderly with two cases of cataract in our study shows the important effect of infection duration or age related $H$. pylori infection. To find the long time effect of infestation or rule of age on $\mathrm{H}$. pylori infection, there is need to do comparative or a large sample study.

\section{REFERENCE}

Banić M, Franceschi F, Babić Z, Gasbarrini A (2012). Extragastric manifestations of Helicobacter pylori infection. Helicobacter $17:(S 1): 45-55$.
Choi CY, Kim MS, Kim JM, Park KH, Hong C (2010). Association between Helicobacter pylori infection and Posner-Schlossman syndrome. Eye 24 (1): 64-69.

Deng B, Li Y, Zhang Y, Bai L, Yang P (2013). Helicobacter Pylori Infection and Lung Cancer; A Review of an Emerging Hypothesis. Carcinogenesis [Epub ahead of print]

Dzintra K, Vētra J (2011). Variation in some anthropometrical parameters of the women with the different iris color in Lativa, Papers on Anthropology XX. pp. 160-170.

Farooq MU, Bhatt A (2008). Helicobacter pylori: Neurological and Ophthalmological Disorders. Internet J. Neurol. 9(2):7.

Feghhi M, Hajiani E, Khataminia G, (2008).Incidence of Helicobacter pylori in central serous chorioretinopathy; a case control study. Jundishapur J. Microbiol. 1(1): 15-19.

Hashemi H, KhabazKhoob M, Yekta AA, Mohammad K, Fotouhi A (2010). Distribution of Iris Colors and its Association with Ocular Disorder in the Tehran Eye Study. Iran. J. Ophthalmol. 22(1):7-14.

Izzotti A, Saccà SC, Bagnis A, Recupero SM (2009). Glaucoma and Helicobacter pylori infection; correlations and controversies. Br. J. Ophthalmol. 93(11):1420-1427.

Jannis K, Nikolaos M, Anastasios GPK, Christos Z, Dimitrios C, Anna B (2003). Increased levels of Helicobacter pylori IgG antibodies in aqueous humor of patients with primary open-angle and exfoliation glaucoma. Graefes Arch. Clin. Exp. Ophthalmol. 241(11): 884-890.

Kountouras J, Zavos C, Sakkias G, Deretzi G, Venizelos I, Arapoglou S, Polyzos SA (2011). Helicobacter pylori infection as a risk factor for both primary open-angle glaucoma and pseudoexfoliative glaucoma in Thessaloniki Eye Study. Am. J. Ophthalmol. 152(6):1079-80.

Kurtz S, Regenbogen M, Goldiner I, Horowitz N, Moshkowitz M(2008). No Association Between Helicobacter pylori Infection or CagAbearing Strains and Glaucoma. J. Glaucoma 17( 3): 223-226.

Morrison PJ (2010). The iris - a window into the genetics of common and rare eye diseases. Ulster Med. J. 79(1):3-5.

Sergio CS, Antonio P, Gian MV, Guido P, Antonio M, Franco B (2006). Prevalence and Treatment of Helicobacter pylori in Patients with Blepharitis. Invest. Ophthalmol. Vis. Sci. 47 (2): 501-508. 